Proceedings of ISFA2016

2016 International Symposium on Flexible Automation

Cleveland, Ohio, USA, 1-3 August, 2016

\title{
LOCALIZING OPERATORS IN THE SMART FACTORY: A REVIEW OF EXISTING TECHNIQUES AND SYSTEMS
}

\author{
Anna Syberfeldt \\ Mikael Ayani \\ Magnus Holm \\ University of Skövde \\ PO-408, Skövde, Sweden \\ anna.syberfeldt@his.se \\ mikael.ayani@ his.se \\ magnus.holm@his.se
}

\author{
Lihui Wang \\ Royal Institute of Technology \\ 10044 Stockholm, Sweden \\ lihuiw@kth.se
}

\author{
Rodney Lindgren-Brewster \\ Volvo Cars Engine \\ Skövde, Sweden \\ rodney.lindgren.brewster@volvocars.com
}

\begin{abstract}
The aim of this paper to give a comprehensive overview of existing techniques and state-of-the-art systems for indoor localization that could be adopted in smart factories of the future. We present different techniques for calculating the position of a moving object using signal transmission and signal measurement, and compare their advantages and disadvantages. The paper also includes a discussion of various localization systems available in the market and compares their most important features. It ends with a discussion of important issues to consider in future work in order to fully implement indoor, real-time localization of operators in the smart factory.
\end{abstract}

\section{INTRODUCTION}

The term "smart factory" refers to the fourth industrial revolution (also called Industry 4.0) and a groundbreaking technological evolution towards cyber-physical systems [1]. With smart factories comes a paradigm shift from centralized to decentralized production which is enabled through the concept of the "Internet-of-Things" [2]. The Internet-of-Things basically means that all objects in a factory (such as machines, tools, products, and human operators) are connected to the Internet and share information with each other [3]. When all objects are online, entirely new possibilities emerge for developing intelligent and adaptive IT-based support systems that can make shop floor operators more flexible and efficient. For example, instead of using traditional, static user interfaces it becomes possible to dynamically download the user interface from the Internet and adapt the information content based on the operator's location. This requires that the positions of all operators are tracked in real-time, which creates a requirement for efficient localization systems. Localization systems have historically been used mainly for outdoor applications (such as GPS-based car navigation), but in the last few years a greater focus on indoor applications has arisen. The aim of this paper to provide a comprehensive overview of existing techniques and state-of-theart systems for indoor localization that could be adopted in the smart factories of the future.

The paper starts by describing different techniques for calculating the position of a moving object using signal transmission and signal measurement. Basic to all of them is that the object carries a signal transmitter and continuously generates a signal that is picked up by a signal measurement unit that calculates the distance to the source of the signal. The position is used in the localization system along with spatial and contextual information about the environment and the objects in it to create a map of the location of each individual object in a building. The main features of the localization systems available in the market are outlined in the paper. The systems implement different techniques for signal transmission and signal measurement, which provide each of them with unique characteristics and thus different advantages and disadvantages. The paper ends with a discussion of important issues to consider in future work in order to fully implement indoor, real-time localization of operators in a smart factory.

\section{SIGNAL TRANSMISSION}

This chapter describes the techniques most commonly used for signal transmission, which is implemented in a hardware unit that is carried by the object to be localized (in this case, an operator). 


\section{Received Signal Strength Indication (RSSI)}

RSSI is based on the assumption that the greater the distance between two nodes, the weaker their relative received signals [4]. This relation is expressed using the formula in Eq. (1)

$P_{r}(d)=P_{0}-10 y \log _{10} d+S$

where $P_{r}$ is the received signal power $(\mathrm{dBm}), \mathrm{P}_{0}$ is the received signal power at one meter's distance $(\mathrm{dBm})$, y is the path-loss exponent (typically 2 to 6 ), $\mathrm{d}$ is the separation between nodes (m), and $\mathrm{S}$ is the large-scale fading variation $(\mathrm{dB})$. The main advantage of RSSI is that the nodes do not have to be time-synchronized, while the main disadvantage is that it cannot be used when there are blocking or shadowing elements such as walls, furniture, or people.

\section{Time of Arrival (ToA)}

In ToA, the arrival time of the signal from the signal transmitter unit and at least three known nodes is measured [5]. By considering the nodes as the center and the distance as the radius of a circle, three circles are defined. The object is then assumed to be located at the intersection of the circles. An example is show in Figure 1 in which A, B, and C are nodes and $\mathrm{P}$ is the object to be positioned. Basically, ToA is the same technique that is used in GPS systems. The main advantages of ToA are that it has high precision and is based on an easy formula. The main drawback is that it assumes that all nodes and the object are time-synchronized. If they are not synchronized the calculation will be inaccurate, even with a few milliseconds of mismatch.

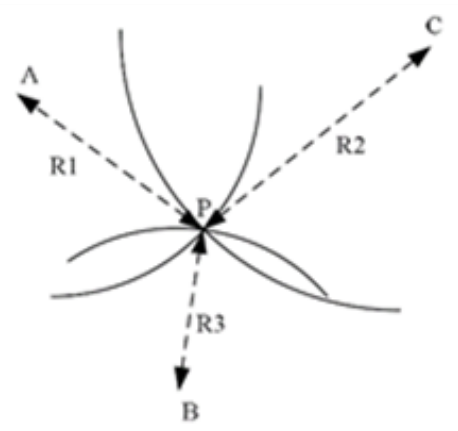

Figure 1. ToA measurement

\section{Time Difference of Arrival (TDoA)}

The basic principle used in TDoA is the same as in ToA: measuring distance by measuring the arrival time of a signal [5]. The main difference between ToA and TDoA is that in the former the object and the nodes need to be synchronized with each other, while in the latter only the nodes have to be synchronized. In TDoA, the time of arrival of the signal to at least three synchronized nodes are measured and hyperbolas are obtained by comparing the time difference between each pair of nodes, as shown in Eq. (2) and Figure 2. The optimal solution of the equations is the position of the target.

$\left\{\begin{array}{l}\sqrt{\left(x_{2}-x_{0}\right)^{2}+\left(y_{2}-y_{0}\right)^{2}}-\sqrt{\left(x_{1}-x_{0}\right)^{2}+\left(y_{1}-y_{0}\right)^{2}}=v \Delta t_{21} \\ \sqrt{\left(x_{3}-x_{0}\right)^{2}+\left(y_{3}-y_{0}\right)^{2}}-\sqrt{\left(x_{1}-x_{0}\right)^{2}+\left(y_{1}-y_{0}\right)^{2}}=v \Delta t_{31}\end{array}\right.$

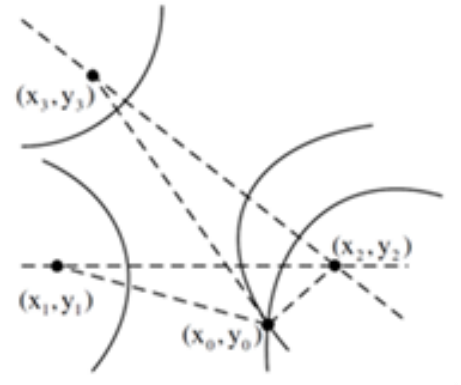

Figure 2. TDoA measurement

The advantage of TDoA compared to ToA is that the synchronization process is easier since only the nodes have to be synchronized. The drawbacks are that it still requires synchronization and that the equation used is non-linear and thus more complex to solve.

\section{Reflectron Time of Flight (RToF)}

RToF measures the time it takes for the signal to travel from the transmitter to the measuring unit and back again [6]. The physical principle applied is the same as in ToA/TDoA. The calculation is initiated when a node $\mathrm{A}$ transmits a signal to another node B. After a known time delay, node B transmits an acknowledgment signal to node A. The time-of-flight is calculated using the formula in Eq. (3)

$$
t_{R T}=2 t_{p}+t_{d}
$$

where $t_{R T}$ is the round-trip time, $t_{p}$ is the time-of-flight, and $t_{d}$ is the time delay. The main advantage of RToF is that it has the same high precision as ToA/TDoA but does not require synchronization. The disadvantage of RToF is that, compared to other techniques, it is highly time consuming since the signal has to travel twice between the sender and the receiver and time delays are included. This means that the application of the technique is limited to scenarios in which the target is not moving fast and where there is considerable time available for calculation.

\section{Angle of Arrival (AoA)}

AoA is based on basic geometry: the direction of an arriving signal is used to calculate an angle that is in turn used to determinate the distance between the transmitter and the target [7]. By finding the intersection of multiple pairs of angle direction lines, the position of the target can be calculated easily and accurately. Only two signals are necessary to calculate a position in two dimensions. An example is shown in Figure 3.

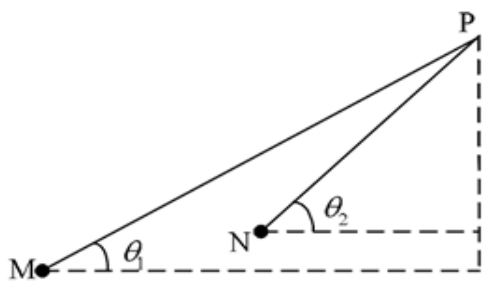

Figure 3. AoA measurement

The advantages of AoA are that it does not require synchronization and that it requires only measurements made at two units to calculate a position. The disadvantages are that the 
precision of the calculation degenerates with the speed of the object and that the measuring unit is negatively affected by shadowing elements. As a result, AoA works best when there is a short distance between the signal transmitter and the signal measurement unit.

\section{Inertial Measurement}

The principle applied in this technique is to calculate changes in rotational attributes like pitch, roll, and yaw [8]. If the initial position of the target is known (which is assumed), the new position of the target can be calculated by measuring the changes. Inertial measurement units (IMUs) are used for inertial measurements. IMUs are electronic devices that measure orientation and gravitational forces using a combination of different solutions such as accelerometers, E-compasses and gyroscopes. The main advantage of the technique is that there is no need to communicate with nodes or other devices to determine a position. The main disadvantage is that the initial position of the object must be known, which means that the technique must be combined with some other technique to be useful. Another disadvantage is that the position is calculated based on the previous measured position, and since each calculation includes a small error, the error will grow over time.

\section{Magnetic Field Measurement}

Earth's geomagnetic field varies from place to place, and the concrete and steel of a building also generate specific fluctuations in magnetic fields [9]. The non-uniform magnetic field can be observed and used to generate a map of the indoor environment that can in turn be used to determine the position of the measuring unit. The main advantages of the technique are that is does not need any geometrical calculation or any synchronization between different devices. The main disadvantage is that the precision is significantly reduced in large, open buildings as such buildings generate quite uniform magnetic fields.

\section{SIGNAL MEASUREMENT}

Just as there are several ways of transmitting signals, so there are several different techniques for measuring signals. The most commonly used are described below.

\section{Radio Frequency (RF)}

There are several different techniques for RF-based measurement, and most of them use fingerprinting [10]. The basic concept of fingerprinting is that a database is built with reference locations inside the building. Regression techniques are then used to match a measurement at a location to the database in order to infer the current position. Figure 4 shows an example of a fingerprint. The bright red spot represents the location of a transmitter. As the distance between receiver and transmitter increases, the signal strength decreases (cooler colors). The fingerprinting process consists of two stages; offline stage and online stage. In the offline stage, the database is created and some reference points from the building are saved in the database. In the online stage, as a mobile unit navigates inside the building, the measurements are compared with the references saved in the database. There are several different RF-based measurement techniques. The most commonly used include Wi-Fi, Bluetooth, RFID, ZigBee, and UWB.

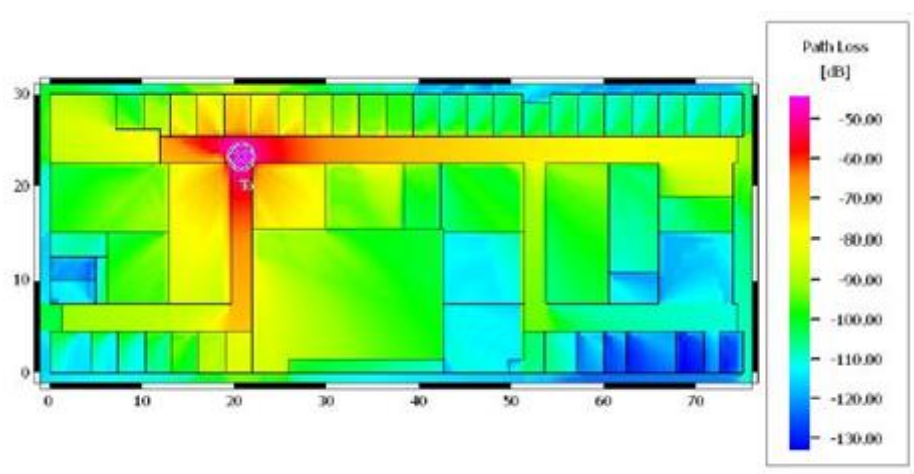

Figure 4. Office fingerprint

$W i-F i$

Wi-Fi is based on the IEEE 802.11 standard and operates mostly at a frequency of $2.4 \mathrm{GHz}$ [11]. The range is approximately 50 to 100 meters with a precision of 1 to 50 meters and an update rate of a few seconds. The RSSI technique is generally used for signal transmission when Wi-Fi is used. The main advantage of $\mathrm{Wi}-\mathrm{Fi}$ is that it is commonly used for network access and therefore it is seldom necessary to invest in or install new hardware. The main drawback of $\mathrm{Wi}-\mathrm{Fi}$ is a relatively poor precision in many indoor environments because the RSSI technique works poorly when there are blocking or shadowing elements.

\section{Bluetooth}

Bluetooth operates in the $2.4 \mathrm{GHz}$ industrial, scientific, and medical (ISM) band and also uses RSSI. It has, however, a shorter range than $\mathrm{Wi}-\mathrm{Fi}$, typically 10 to 15 meters [12]. The main advantage of Bluetooth is that the technique is already integrated in common off-the-self devices such as mobile phones and tablets. Other advantages are small size and low power consumption. The main disadvantages of Bluetooth are that it is easily affected by noise and that it might be unstable in complex spatial environments.

\section{Radio Frequency Identification (RFID)}

With RFID, electromagnetic transmission is used to retrieve and store data in an RF-compatible integrated circuit, often called a "tag" [13]. RFID tags are either passive or active. Passive tags operate without a battery and can be read from between 0 and 10 meters away. Active RFID tags are battery-powered and have a reading range between 0 and 50 meters. Most commonly RFIDbased positioning is implemented using a system called LANDMARC [14]. LANDMARC is based on RSSI. To increase the precision without increasing the number of readers, the system uses active RFID as base stations and for fixed location references. The main advantage of using RFID is that passive tags are very cheap. A disadvantage is that the precision of the positioning is highly dependent on the number of base stations and reference points.

\section{ZigBee}

ZigBee is an emerging standard for wireless communication that is designed for scenarios with low data load when low power consumption is needed [15]. ZigBee usually uses RSSI for positioning. The range in indoor environments is typically 20 to 30 meters. The main advantages of ZigBee are low power consumption and small size. The main drawback of ZigBee is that 
it can cause, and suffer from, interference from other devices using the same radio frequency.

\section{Ultra-wideband (UWB)}

UWB is a radio technology for short-range, high-bandwidth communication with a strong multipath resistance [16]. The spectrum used is wide, ranging from 3.1 to $10.6 \mathrm{GHz}$. UWB commonly uses short pulses (typically having a duration of nanoseconds) and thus spreads the spectrum of the transmitted signal over a wide frequency. The precision of UWB is approximately 15 centimeters, which provides very good precision compared to other RF-based techniques. Another advantage of UWB is that it can be used close to other RFdevices without causing or suffering from interference thanks to the differences in signal types and radio spectrum used. A disadvantage of UWB is that it is expensive.

\section{Infrared Radiation (IR)}

IR uses non-visible light to implement communication between two nodes [17]. The technique is widely used in wireless communications and available in many consumer products, for example mobile phones and TVs. Most IR-based devices use socalled "line-of-sight communication" between the transmitter and the receiver. With IR, a precision of a few centimeters can be obtained. A further advantage of IR, besides high precision, is that the device is small and lightweight. The disadvantages of IR include the requirement of an unobstructed line-of-sight between the transmitter and the receiver, that the range is quite small (a few meters), and that it is affected by interference from strong light sources.

\section{Ultrasound}

Ultrasound is based on the same principle as used by bats for navigation, namely sound waves with very high frequencies [18]. Distance is estimated by measuring the time it takes for the ultrasound signal to travel from the transmitter to a receiver. A precision of a few centimeters can be obtained with ultrasound, which is a clear advantage of the technique. The main disadvantage is that, as with IR, line-of-sight is needed between the transmitter and the receiver. Another disadvantage is that the technique suffers from interference from ultrasound signals generated by other sources.

\section{Summary of comparison}

Table 1 summarizes the most important characteristics of the signal measurement techniques. The summary allows the different techniques to be compared in order to select the optimal technique for a specific scenario.

The table shows that the techniques have quite different characteristics and thus different advantages and disadvantages. None of the techniques is universally superior to the others, which becomes evident when investigating the various indoor localization systems that are available on the market.

\section{EXISTING SYSTEMS FOR INDOOR LOCALIZATION}

The most well-known systems for indoor localization all have unique implementations and use different techniques for signal transmission and signal measurement. The description of these systems is followed by a summarized comparison between them.
Table 1. Comparison of signal measurement techniques

\begin{tabular}{|c|c|c|c|c|}
\hline Technique & Precision & $\begin{array}{c}\text { Signal } \\
\text { transmission }\end{array}$ & $\begin{array}{c}\text { Power } \\
\text { consumption }\end{array}$ & Cost \\
\hline Wi-Fi & $1-5 \mathrm{~m}$ & $\begin{array}{c}\text { ToA, TDoA, } \\
\text { RSSI }\end{array}$ & High & Low \\
\hline Bluetooth & $2-5 \mathrm{~m}$ & RSSI & Low & High \\
\hline RFID & $1-2 \mathrm{~m}$ & $\begin{array}{c}\text { ToA } \\
\text { RSSI }\end{array}$ & Low & Low \\
\hline ZigBee & $3-5 \mathrm{~m}$ & RSSI & Low & Low \\
\hline UWB & $\begin{array}{c}15 \mathrm{~cm}-1 \\
\mathrm{~m}\end{array}$ & $\begin{array}{c}\text { ToA, TDoA, } \\
\text { AoA }\end{array}$ & Medium & High \\
\hline IR & $\begin{array}{c}10 \mathrm{~cm}-2 \\
\mathrm{~m}\end{array}$ & ToA & Low & Medium \\
\hline Ultrasound & $3 \mathrm{~cm}-1 \mathrm{~m}$ & ToA, AoA & Low & Medium \\
\hline
\end{tabular}

\section{SenionLab}

The SenionLab system (www.senionlab.com) is based on common technologies available in smart phones, including IMU, Wi-Fi, and Bluetooth, in combination with Senion beacons, which use the iBeacon technology from Apple. About one beacon per 100 square meters is needed. In this system, an initial position for an object is first determined using Wi-Fi and Bluetooth. Once the initial position is known, the system switches to using IMU to track the movement of the object and thus its position. The location of the object is computed locally in an on-board device.

\section{Aruba NetWorks}

The Aruba NetWorks system (www.arubanetworks.com) is mainly intended to be used to detect the presence of smart phones in a specific area and to push information to them. Typical application domains include shopping malls and sports arenas. The system uses low energy Bluetooth beacons called Aruba beacons and Wi-Fi devices that are spread out in the area. Besides detecting smart phones, these can also be used to calculate their positions by measuring the signal strength between the phone and each beacon/device.

\section{Locata}

The Locata system (www.locata.com) uses a so-called Pseudolite network and a radio frequency method commonly used in satellite navigation. The Locata system uses two devices, a transceiver and a receiver. A patented wireless synchronization technology called TimeLoc is used for synchronization. This technology allows Locata to broadcast GPS-like signals via a synchronous, fully autonomous, and self-initializing groundbased network. The synchronization between devices takes place within a nanosecond and virtually exact positioning can be provided. However, this very high quality comes at a very high price.

\section{BlinkSight}

The BlinkSight system (www.blinksight.com) performs localizations using a special microchip that combines digital processing elements and UWB technology. The system operates in both the 3.1 to $4.8 \mathrm{GHz}$ and the 6 to $10 \mathrm{GHz}$ UWB band and can thus co-exist with other wireless technologies. The input 
voltage range of the microchip is 1.5 to $3.6 \mathrm{~V}$, which makes it possible to integrate the chip in different devices such as tags, base stations, and mobile phones. The chip continuously communicates with a base station that is connected to a server. Special software runs on the server to derive the position of each device. The precision of the localization is within 10 centimeters in line-of-sight and about 50 centimeters otherwise.

\section{LIPS}

The LIPS system (http://lips.si/) uses radio frequency signals at $2.45 \mathrm{GHz}$ to localize objects. The system includes three different devices; tags, anchors, and access points. The tag is placed on the object to be localized, and it communicates with nearby anchors, which in turn communicate with the closest access point. The range of the devices is up to 30 meters and the precision of the positioning is approximately 1 meter, depending on environmental conditions and the positioning of the anchors. The system can co-exist with other radiofrequency technologies without interference.

\section{Ubisense}

The Ubisense system (http://ubisense.net/) uses UWB for localization. In the system, an active tag is carried by the object to be localized, and this tag sends signals to sensors that are distributed in the area. All sensors must be wired to be accurately synchronized with each other. The sensors include software that calculates the position of tags several times per second. The precision obtained is about 15 centimeters.

\section{Ekahau}

The Ekahau system (www.ekahau.com) is based on Wi-Fi and RFID. Already existing Wi-Fi infrastructure can be used in order to reduce the implementation cost. The precision obtained with the system is about 5 to 15 meters in an open area and 1 to 3 meters in smaller rooms. Increased precision in delimited areas can be obtained using a special device called Ekahau LB2, which is based on IR technology. With this device, the precision can be improved to about 1 meter. The device is battery-powered and can therefore be placed anywhere without wiring.

\section{IndoorAtlas}

The IndoorAtlas system (www.indooratlas.com) performs localizations based on Wi-Fi, Bluetooth, and magnetic field measurements. The system, which can be implemented in any modern smart phone that has a compass, measures the geomagnetic field and sends the information to a central server that calculates the position. The precision of the position is highly dependent on the surrounding environment. In buildings with many walls and an abundance of concrete and steel, the precision can be within 10 centimeters, while in buildings with large open spaces the precision decreases significantly since there are no substantial differences in the magnetic field inside the building.

\section{InfSoft}

The InfSoft system (www.infsoft.com) is based on Wi-Fi and Bluetooth and utilizes devices already present in the building where the system to be used. In cases where there are no, or few, Wi-Fi or Bluetooth devices in the building, the company offers special Bluetooth beacons to be used with the system. The precision of the system is about 1 to 4 meters.

\section{Summary of comparison}

Table 2 provides a comparative overview of the most important features of current indoor localization. Column 3 in the table, Precision, should be interpreted in the following way:

- Very high $=$ precision within $0-10 \mathrm{~cm}$ centimeters

- $\mathrm{High}=$ precision within 10-50 centimeters

- $\quad$ Medium $=$ precision within 0.5-1 meter

- $\quad$ Low $=$ precision within \pm 1 meter

From Table 2, and from the description of the systems, it is clear that the systems are highly diverse. They each have unique implementations and use different techniques for signal transmission and signal measurement. It is impossible to choose a single best system since the strengths and weaknesses of the systems depend entirely on the intended application in combination with the physical circumstances in the building and the investment budget.

Table 2. Comparison of indoor localization systems

\begin{tabular}{|l|l|l|l|l|}
\hline System & Technique & Precision & $\begin{array}{l}\text { Develop- } \\
\text { ment kit }\end{array}$ & Cost \\
\hline SenionLab & $\begin{array}{l}\text { Wi-Fi } \\
\text { Bluetooth } \\
\text { IMU }\end{array}$ & Medium & Yes & Low \\
\hline $\begin{array}{l}\text { Aruba } \\
\text { Network }\end{array}$ & $\begin{array}{l}\text { Bluetooth } \\
\text { Wi-Fi }\end{array}$ & Low & No & Low \\
\hline Locata & RF & Very high & No & $\begin{array}{l}\text { Very } \\
\text { expensive }\end{array}$ \\
\hline BlinkSight & UWB & High & No & Medium \\
\hline LIPS & RF & Medium & Yes & Expensive \\
\hline Ubisense & UWB & High & Yes & Medium \\
\hline Ekahau & $\begin{array}{l}\text { Wi-Fi } \\
\text { RFID }\end{array}$ & Low & Yes & Low \\
\hline $\begin{array}{l}\text { Magnetic } \\
\text { Atlas }\end{array}$ & $\begin{array}{l}\text { Wi-Fi } \\
\text { Bluetooth }\end{array}$ & Medium & Yes & Very Low \\
\hline InfSoft & $\begin{array}{l}\text { Wi-Fi } \\
\text { Bluetooth }\end{array}$ & Low & Yes & Low \\
\hline
\end{tabular}

\section{CONCLUSIONS}

There are several commercial indoor localization systems in the market designed for different purposes and based on different technologies. A review of these systems shows that none of them are tailor-made for shop floors or explicitly tackle all the environmental challenges present in factories. Such challenges include large spaces with high ceilings, numerous metal objects (e.g. machines, racks, work benches, pallets, and objects within walls), dirt, dust, many machines communicating wirelessly, and a constantly changing environment due to moving objects. The existing systems are mainly for other types of environments and applications, such as locating patients in a hospital, tracking valuable tools at a construction site, customer navigation in shopping malls, and navigation of mobile robots in dangerous environments. In order to successfully develop position-based support systems for shop floor operators and fully utilize the potential of indoor localization in a smart factory, a new system 
tailor-made for industrial shop floors must be developed. A number of requirements for such system have been identified by the authors and are described below.

\section{Requirements related to the physical environment}

The system must work efficiently under the following conditions, which are typical of industrial environments:

- Very large buildings.

- Mixed layout with highly diverse spaces ranging from spacious halls to small rooms.

- Many objects and many walls (shadowing elements).

- Numerous metal objects such as machines, racks, work benches, pallets, and walls.

- Dirt and dust.

- Varying temperatures.

Requirements related to functionality

The system must be able to:

- Handle a very large number (potentially thousands) of operators working simultaneously.

- Identify a position with high precision, having an error margin of less than 20 centimeters.

- Avoid disturbing existing equipment (such as machines, PLCs, and computers) that communicate wirelessly.

- Efficiently handle an environment that constantly changes due to moving objects.

- Support a wide range of hardware units and several different signal measurement techniques.

- Be flexible with respect to changes in the hardware infrastructure (changing signal transmission units).

- Allow for easy integration of new devices added after the system has been set up.

Requirements related to implementation and deployment The system should:

- Be easy to configure and install (no specific expertise should be needed).

- Have a minimal set-up time (to save time and ensure a low threshold).

- Make use of already installed devices for signal transmission in the factory (such as existing Wi-Fi infrastructure) in order to reduce cost.

- Work with commonly used off-the-shelf hardware (e.g. smartphones) in order to reduce cost.

Requirements related to adoption and future development

To ensure widespread use and a long lifetime, the system should:

- Have a low cost.

- Be available with open software that anyone can modify and improve.

- Be based on general, well-documented signal transmission and signal measurement techniques.

Developing a localization system that fulfills all these requirements will represent an important step towards fully realizing a smart factory.

\section{REFERENCES}

[1] Wang, S., Wan, J., Zhang, D., Li, D. and Zhang, C., 2016, "Towards smart factory for Industry 4.0: A self-organized multi-agent system with big data based feedback and coordination," Computer Networks, ISSN 1389-1286, http://dx.doi.org/10.1016/j.comnet.2015.12.017.

[2] Zuehlke, D., 2010, "SmartFactory - Towards a factory-ofthings," Annual Reviews in Control, 34(1), pp. 129-138.

[3] Shrouf, F., Ordieres, J., Miragliotta, G., 2014, "Smart factories in Industry 4.0: A review of the concept and of energy management approached in production based on the Internet of Things paradigm," IEEE International Conference on Industrial Engineering and Engineering Management, pp. 697-701.

[4] Sauter, M., 2010, From GSM to LTE: An Introduction to Mobile Networks and Mobile Broadband (eBook). John Wiley \& Sons, Chap. 3.7.1. ISBN 9780470978221.

[5] Malik, A., 2009, RTLS For Dummies, Wiley New York 2009

[6] Mamyrin, B. A., Karataev, V. I., Shmikk, D. V., Zagulin, V. A., 1973, "The mass-reflectron, a new nonmagnetic time-offlight mass spectrometer with high resolution," Sov. Phys. JETP 37(45).

[7] Li, M. and Lu, Y., 2008, "Angle-of-arrival estimation for localization and communication in wireless networks," Proc. 16th European Signal Processing Conference, pp.1-5.

[8] Eshbach, O.W. Eshbach's Handbook of Engineering Fundamentals, Byron.

[9] Purcell, E., 2011, Electricity and Magnetism (2nd ed.), Cambridge University Press. ISBN 1107013607.

[10] Zhang, A., Yuan, Y., Wu, Q., Zhu, S. and Deng, J., "Wireless Localization Based on RSSI Fingerprint Feature Vector," International Journal of Distributed Sensor Networks, vol. 2015. DOI:10.1155/2015/528747.

[11] Lim H., Kung L., Hou J.C., Haiyun, L., 2010, "Zeroconfiguration indoor localization over IEEE 802.11 wireless infrastructure," Wireless Networks 16(2), pp.405-20.

[12] Pei, L., Chen, R., Liu, J., Kuusniemi, H., Tenhunen, T. and Chen, Y., 2010, "Using Inquiry-based Bluetooth RSSI Probability Distributions for Indoor Positioning," Journal of Global Positioning Systems (2010) Vol.9, No.2 :122-130. DOI: 10.5081/jgps.9.2.122

[13] Saab, S. and Nakad, Z., 2011, "A Standalone RFID Indoor Positioning System Using Passive Tags," IEEE Transactions on Industrial Electronics, 58(5), pp.1961-1970. doi: 10.1109/TIE.2010.2055774

[14] Ni, L., Yunha, L., Cho, Y. and Patil, A.P., 2003, "LANDMARC: indoor location sensing using active RFID," Proceedings of the First IEEE International Conference on Pervasive Computing and Communications, pp.407-415. doi: 10.1109/PERCOM.2003.1192765

[15] Benkic, K.., Malajner, M., Planinsic, P. and Cucej, Z., 2008, "Using RSSI value for distance estimation in wireless sensor networks based on ZigBee," 15th International Conference on systems, Signals and Image Processing, pp.303-306. doi: 10.1109/IWSSIP.2008.4604427

[16] Wang, S., Waadt, A., Burnic, A., Dong X., Kocks, C., Bruck, G.H. and Jung, P., 2010, "System implementation study on 
RSSI based positioning in UWB networks," 7th International Symposium on Wireless Communication Systems, pp.36-40. doi: 10.1109/ISWCS.2010.5624355

[17] Zahid, F., Nordin, R. and Ismail, M., 2013, "Recent Advances in Wireless Indoor Localization Techniques and System," Journal of Computer Networks and Communications. doi:10.1155/2013/185138

[18] Medina, C., Segura, J.C. and Holm, S., 2012, "Feasibility of ultrasound positioning based on signal strength," International Conference on Indoor Positioning and Indoor Navigation, pp.1-9. doi: 10.1109/IPIN.2012.6418917 